\title{
ANNUAL REPORT \\ TO THE PRESIDENT AND CONGRESS \\ 1977-1978
}

For sale by the Superintendent of Documents, U S Government Printing Otfice Washington, D C 20402 